\title{
Stability of wall-bounded flows using one-way spatial integration of Navier-Stokes equations
}

\author{
Georgios Rigas, Tim Colonius \\ California Institute of Technology, Pasadena, CA, USA \\ Michael Beyar \\ Boeing Research \& Technology, Huntington Beach, CA, USA
}

\begin{abstract}
A method for constructing well-posed one-way equations for calculating disturbances of slowly-varying flows was recently introduced (Towne \& Colonius, JCP, Vol. 300, 2015). The linearized Navier-Stokes equations are modified such that all upstream propagating modes are removed from the operator. The resulting equations, termed one-way NavierStokes equations, are stable and can be solved efficiectly in the frequency domain as a spatial initial value problem in which initial perturbations are specified at the domain inlet and propagated downstream by spatial integration. To date, the method has been used to predict large-scale wavepacket structures and their acoustic radiation in turbulent jets. In this paper, the method is extended and applied to wall-bounded flows. Specifically, we examine the spatial stability of two- and three-dimensional boundary layers, corresponding to the Blasius and the Falkner-Skan-Cooke flows, and predict the evolution of unstable Tollmien-Schlichting waves and crossflow vortices, respectively. The method is validated against well-known results from the literature.
\end{abstract}

\section{Introduction}

Hydrodynamic stability analysis is a critical tool for transition prediction in the laminar regimes, and, when applied to the mean of a turbulent mean flow has been used to predict coherent structures in some flows. ${ }^{1-3}$ In either case, the analysis can provide significant insight for developing and implementing flow control techniques. For laminar flow control, the theory identifies how instabilities can be delayed; in separation control, the theory identifies how small actuator inputs can be amplified; in turbulent drag control it identifies dynamically significant structures that should be targeted by control.

Stability tools have been successfully applied to canonical two-dimensional (2D) flows over the last years. However, practical flows of relevance to the aviation industry are often three-dimensional (3D). For 3D flows questions of stability, receptivity, secondary flows, and coherent structures, require the solution of large $3 \mathrm{D}$, partial-derivative eigenvalue problems. Reduced-order approximations are thus required for engineering prediction. A regularization of the equations of motion sometimes permits a very fast spatial marching procedure that results in a huge reduction in computational expense. One such approach is the so-called parabolized stability equations ${ }^{5}$ (PSE). An advantage of the PSE over the local stability theory (LST) is that the base flow and disturbances are assumed to evolve slowly in the dominant direction of the flow, which allows important weakly nonparallel effects to be captured. Because of its computational efficiency, PSE forms the basis of many advanced transition prediction schemes, and it has been extended to fully $3 \mathrm{D}$ flows. Nonlinear extensions of the method allow for the interaction of finite-amplitude disturbances, and the creation of secondary flows through mean-flow deformation. However, PSE often fail to converge, especially in flows that exhibit multiple unstable modes or modes that span multiple length scales.

Recently a novel one-way spatial marching algorithm has been developed, ${ }^{8-10}$ known as one-way Euler (OWE), and applied to shear flows governed by the Euler and Navier-Stokes equations. The one-way marching method yields a coupled system of algebraic and ordinary differential equations that evolve in the dominant flow direction and that can be solved in a small fraction of the time required to integrate the $2 \mathrm{D}$

*rigas@caltech.edu 
and 3D equations. The new method overcomes the principle flaw in PSE, namely the ad hoc regularization that removes upstream propagating modes. Instead of regularizing the equations, the one-way method correctly parabolizes them in a computationally efficient way. The underlying principle is the estimation of the local spectrum in any cross-stream plane and the design of an efficient spectral filter that eliminates modes with upstream group velocity. The resulting equations are convergent for convectively unstable flows, including free shear flows (jets, mixing layers) and boundary-layer flows. To date, the method has been used to study large-scale wavepacket structures in round high-speed turbulent jets. These structures are relevant to the dominant aft angle noise radiation in jets, but the multi-scale nature of acoustic radiation from these wavepackets leads to either instability or excessive damping in PSE predictions.

In this paper, we extend the one-way methodology to the Navier-Stokes equations for studying the stability of wall-bounded flows. In section II we present the governing equations and details of the method. In section III, we demonstrate and validate the ability of the one-way Navier-Stokes (OWNS) equations to accurately capture spatial modal instabilities of canonical 2D and 3D boundary-layer flows. Finally, conclusions are drawn in section IV.

\section{Method}

We begin with the compressible Navier-Stokes equations written in Cartesian coordinates in terms of specific volume $\nu$, velocity $\mathbf{u}=\left(u_{x}, u_{y}, u_{z}\right)$ and pressure $p$ :

$$
\begin{aligned}
& \frac{D \nu}{D t}-\nu(\nabla \cdot \mathbf{u})=0, \\
& \frac{D \mathbf{u}}{D t}+\nu \nabla p=\frac{1}{R e} \nu \nabla^{2} \mathbf{u}, \\
& \frac{D p}{D t}+\gamma p(\nabla \cdot \mathbf{u})=\frac{\gamma}{\operatorname{Pr} R e}\left(\nu \nabla^{2} p+p \nabla^{2} \nu\right) .
\end{aligned}
$$

The above equations have been non-dimensionalized by an ambient speed of sound and density, and a problem dependent length scale. The fluid is approximated as a perfect gas with specific heat ratio $\gamma$ and constant Reynolds number Re and Prandtl number Pr. For convenience, we have assumed that the gradient of dilation is small and viscous dissipation has been neglected in the present analysis.

The flow field $\mathbf{q}=\left(\nu, u_{x}, u_{y}, p\right)^{T}$ is decomposed into a steady baseflow $\overline{\mathbf{q}}$ and unsteady fluctuations $\mathbf{q}^{\prime}$ :

$$
\mathbf{q}(x, y, z, t)=\overline{\mathbf{q}}(x, y, z)+\mathbf{q}^{\prime}(x, y, z, t) .
$$

Here, $x, y, z$, correspond to the streamwise, transverse and spanwise directions, respectively. If non-linear products of fluctuations are neglected, the fluctuating field satisfies the linearized compressible Navier-Stokes equations:

$$
\frac{\partial \mathbf{q}^{\prime}}{\partial t}+\mathbf{A} \frac{\partial \mathbf{q}^{\prime}}{\partial x}+\mathbf{B}_{y} \frac{\partial \mathbf{q}^{\prime}}{\partial y}+\mathbf{B}_{z} \frac{\partial \mathbf{q}^{\prime}}{\partial z}+\mathbf{B}_{x x} \frac{\partial^{2} \mathbf{q}^{\prime}}{\partial x^{2}}+\mathbf{B}_{y y} \frac{\partial^{2} \mathbf{q}^{\prime}}{\partial y^{2}}+\mathbf{B}_{z z} \frac{\partial^{2} \mathbf{q}^{\prime}}{\partial z^{2}}+\mathbf{C q}^{\prime}=0
$$

The matrix coefficients are functions of the baseflow and are given in Appendix A.

The perturbation equations are further simplified by neglecting viscous derivative terms of the perturbation in the streamwise direction. This is in accordance with the unsteady boundary layer equations which are derived by neglecting terms of order $1 / R e^{2}$. As a consequence of this order-of-magnitude analysis, all viscous terms containing derivatives parallel to the wall can be dropped, since they are substantially smaller than viscous terms containing derivatives normal to the wall. Although this is not necessary, it simplifies the process of decoupling the upstream and downstream dynamics contained within the linearized Navier-Stokes operator.

The equations can be written in the frequency domain, by expanding $\mathbf{q}^{\prime}(x, y, z, t)=\sum \hat{\mathbf{q}}_{\omega, \beta}(x, y) e^{-i \omega t+i \beta z}$. Also, the $z$ direction has been assumed to be homogeneous with spanwise wavenumber $\beta$. Then the semidiscretized problem can be expressed as a spatial evolution equation:

$$
\mathbf{A} \frac{\partial \hat{\mathbf{q}}}{\partial x}=\left(i \omega-\mathbf{B}_{y} \mathbf{D}_{y}-i \beta \mathbf{B}_{z}-\mathbf{B}_{y y} \mathbf{D}_{y y}+\beta^{2} \mathbf{B}_{z z}-\mathbf{C}\right) \hat{\mathbf{q}}:=\mathbf{M} \hat{\mathbf{q}}
$$

with $\mathbf{D}_{y}$ and $\mathbf{D}_{y y}$ being the differentiation matrices in the transverse direction. At this stage, boundary conditions are also incorporated into $\mathbf{B}_{y}$. 


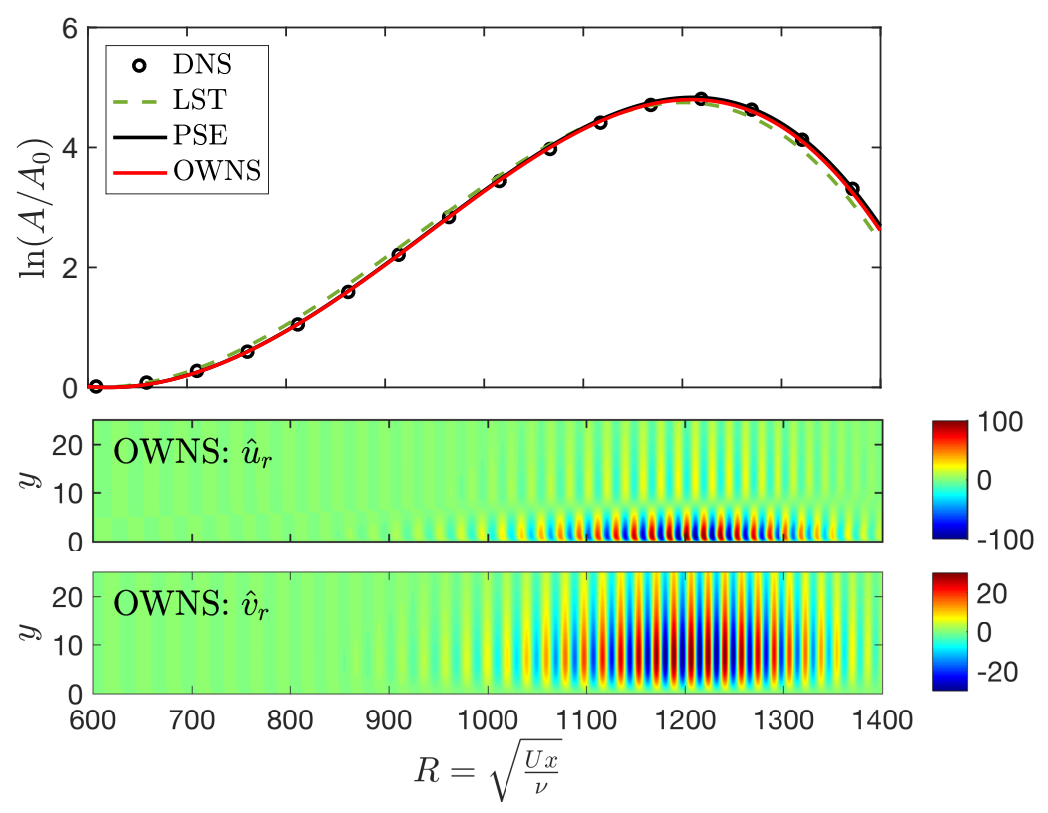

Figure 1. Tollmien-Schlichting instability of the Blasius flow for $F=40 \cdot 10^{-6}$ and Mach number $M \ll 1$. Amplitude growth curves based on $\hat{u}_{\max }$ (top) and eigenfunctions of the $2 \mathrm{D}$ perturbation corresponding to the real part of the streamwise and transverse velocity (middle and bottom). Comparison of LST, PSE and OWNS. DNS data obtained from literature. ${ }^{6}$

Upstream propagating waves supported by equation (4) are formally removed using the one-way method, without analytically factorizing the dispersion relation, resulting in equations which are well-posed and can be solved by spatial marching. Details for the theoretical framework and implementation of the method can be found in the references. ${ }^{8-10}$ It should be emphasized that this is significantly different than other methods, i.e. PSE, which achieve a stable spatial march by numerically damping upstream propagating waves, either by using an implicit axial discretization along with a restriction on the minimum step size ${ }^{11}$ or by explicitly adding damping terms to the equations. ${ }^{12}$ The damping prevents the upstream waves from destabilizing the spatial march, but also has the unintended consequence of damping, to different degrees, all of the downstream waves.

\section{Validation}

The OWNS will be demonstrated by examining the spatial stability of canonical wall-bounded flows. These correspond here to the 2D Blasius flow and the 3D Falkner-Skan-Cooke flow. The method will be validated against predictions from LST and PSE, and direct numerical simulation (DNS) results obtained from the literature.

The OWNS equations are discretized in the transverse direction using fourth-order central finite differences with summation-by-parts boundary closure. Far-field radiation boundary conditions are enforced at the free transverse boundary using a super-grid damping layer ${ }^{13}$ truncated by Thompson ${ }^{14}$ characteristic conditions. Isothermal no-slip boundary conditions $(\hat{u}=\hat{v}=\hat{w}=\hat{T}=0)$ are enforced on the wall. ${ }^{15}$ The OWNS equations are integrated in $x$ using a backward differentiation formula of order 2 (BDF2).

\section{A. 2D Blasius boundary layer}

In this section we examine the stability of the 2D Blasius flow. A Howarth-Dorodnitsyn transformation is applied to the boundary layer equations and a self-similar solution is obtained. Under this transformation the governing compressible boundary layer equations reduce to a set of ODE's. ${ }^{16}$ All quantities presented in this section are nondimensionalized using the freestream velocity $U_{\infty}$ and the fixed length $\delta_{0}=R_{0}=\sqrt{\nu_{\infty} x_{0} / U_{\infty}}$, where $x_{0}$ is a fixed dimensional distance from the leading edge, here taken to be the starting location of the analysis, and $\nu_{\infty}$ the kinematic viscosity in the freestream. 
In figure 1 we show the amplification of the spatially unstable Tollmien-Schlichting (TS) wave for a fixed nondimensional frequency $F=\omega R=\omega^{*} \nu_{\infty} / U_{\infty}^{2}=40 \cdot 10^{-6}$, where * denotes a dimensional quantity. Results were obtained at the incompressible limit at Mach number $M=0.01$. The PSE results converge to the OWNS ones. Also, LST results show good agreement, despite the assumption of parallel flow. In general, for this type of flow, the nonparallel effects on 2D waves are small and decrease with frequency, as has been shown previously.
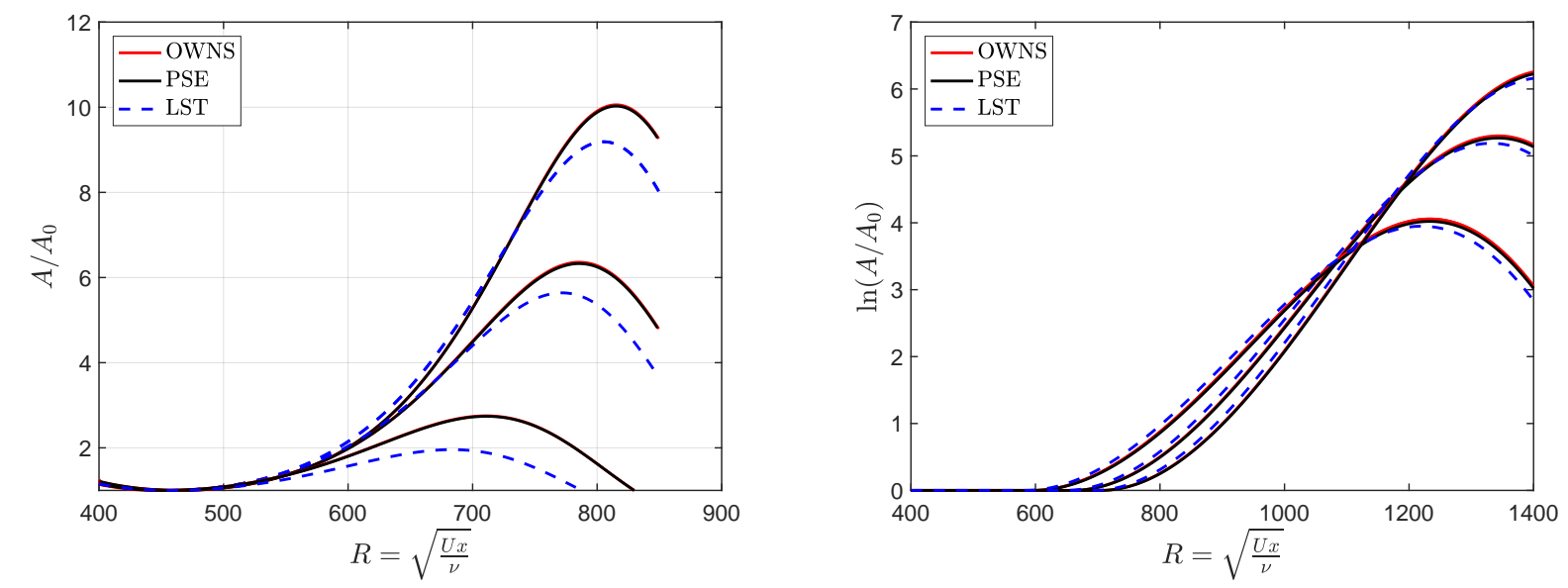

Figure 2. Amplitude growth curves of TS waves based on $\hat{u}_{\max }$. Left: Oblique 3D waves with $F=86 \cdot 10^{-6}$ and $M \ll 1$ for different spanwise wavenumbers $\beta$. Results are shown in terms of the parameter $b=\beta \cdot 10^{3} / R_{0}$ with values $\mathbf{0}, \mathbf{0 . 1}, \mathbf{0 . 2}$ from top to bottom. Right: $2 \mathrm{D}$ waves with $F=40 \cdot 10^{-6}$ for Mach numbers $0.02,0.5,1.0$ from top to bottom. Comparison of LST, PSE and OWNS.

While 2D disturbances indicate a small effect of nonparallelism, the growth of 3D disturbances is substantially different. In figure $2 \mathrm{a}$, we compare the growth rate of $3 \mathrm{D}$ disturbances of the Blasius flow for various spanwise wavenumbers $\beta$. The parameter $b=\beta \cdot 10^{3} / R_{0}$ has been introduced which is independent of the reference scale $\delta_{0}$. These waves propagate in an oblique direction to the freestream. Local theory under predicts their growth noticeably for spanwise wavenumbers corresponding to $b>0.1$. For all cases, the PSE results converge to the OWNS ones.

Finally, the OWNS approach was tested for compressible regimes and the results are shown in figure $2 \mathrm{~b}$, for 2D disturbances with $F=40 \cdot 10^{-6}$. The base-flow was obtained at various Mach numbers for freestream temperature $T_{0}=288 \mathrm{~K}$ and adiabatic wall. The results indicate similar behavior as in the incompressible cases for the range of Mach numbers examined here, $M \leq 1$.

In summary, we have examined the evolution of $2 \mathrm{D}$ and $3 \mathrm{D}$ disturbances of the Blasius flow. It has been extensively shown in the literature that nonparallel effects are significant for 3D disturbances in wall bounded flows. For these cases, where the flow is dominated by a single mode, here TS wave, the PSE method accurately captures the growth of the associated disturbance. In this section we have validated the OWNS approach and we have shown that the PSE results converge to the OWNS ones.

\section{B. 3D Falkner-Skan-Cooke boundary layer}

Boundary layers developing over swept wings are 3D and substantially different from their 2D counterparts, as the ones examined above, due to the existence of crossflow. ${ }^{17}$ The crossflow profile is a result of the pressure gradient and sweep angle which leads to a curved streamline in the outer inviscid flow. The crossflow profile typically exhibits an inflection point, which gives rise to an instability of inviscid type. Crossflow disturbances appear as boundary layer streaks closely following the external streamline. ${ }^{18}$ Swept wing boundary-layer flows are typically unstable to both crossflow and Tollmien-Schlichting waves.

The validation of the OWNS in the following is based on the incompressible Falkner-Skan-Cooke ${ }^{19}$ boundary boundary layer studied by ${ }^{20}$ and ${ }^{21,22}$ which closely mimics swept-wing experiments. ${ }^{23}$ The parameters chosen in these studies were $x_{n}=167, \phi\left(x_{n}\right)=45^{\circ}$ and $m=0.2$. The parameter $m$ is related to the Hartree parameter $\beta_{H}$ through $m=\beta_{H} /\left(2-\beta_{H}\right)$ and determines the pressure gradient. If the chordwise $U_{e}$ and 
spanwise $W_{e}$ velocities of the external inviscid flow obey a power law of the form:

$$
U_{e}=\left(\frac{x}{x_{n}}\right)^{m}, \quad W_{e}=U_{e} \tan \phi,
$$

the similarity solution for a swept flat plate boundary layer is obtained by solving a system of coupled ordinary differential equations for the compressible case. ${ }^{24}$ The compressible baseflow and perturbation equations are solved here at the incompressible limit by setting $M=0.01$.

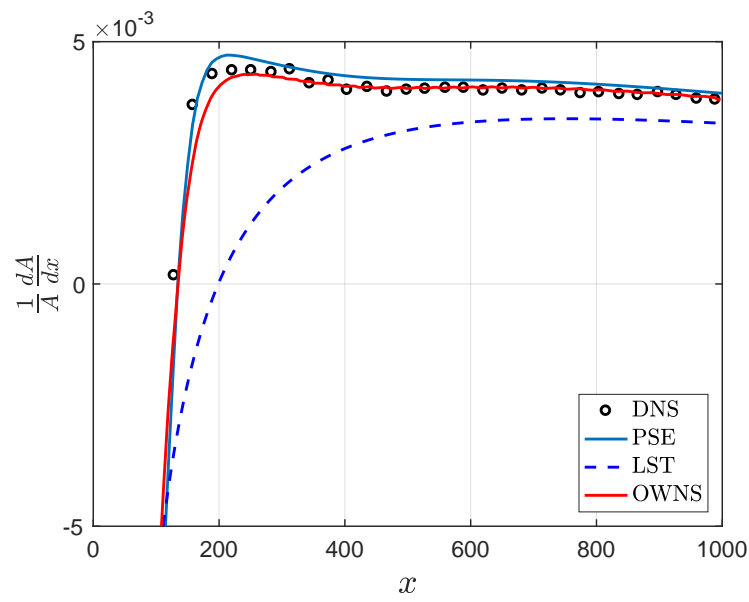

Figure 3. Spatial growth rate of stationary crossflow disturbance with $\beta=0.19, \omega=0$. Accelerating FalknerSkan-Cooke boundary layer with $m=0.2, M \ll 1$. DNS results obtained from. ${ }^{22}$

The spatial growth rates of the unstable crossflow instability obtained from OWNS, PSE and LST are shown if figure 3. Results are obtained for a stationary $(\omega=0)$ crossflow wave with spanwise wavenumber $\beta$ equal to 0.19 and compared with DNS results. ${ }^{21}$ Figure 4 shows the associated dominant 3D velocity component $\hat{u}_{s}$ projected onto the local direction of the external streamline indicating a streaky structure.

As mentioned above, the PSE is often non-convergent due to the minimum step-size restriction, when Euler implicit schemes are employed to numerically damp upstream propagating waves which may cause numerical instability in the scheme. PSE results were obtained here using the minimum step size allowed. It was shown in ${ }^{21}$ that the PSE solution converges to the DNS results only when the disturbance pressure term $d \hat{p} / d x$ is neglected, for the specific case examined here. For the OWNS no such assumption is necessary and the scheme converges to the DNS data.

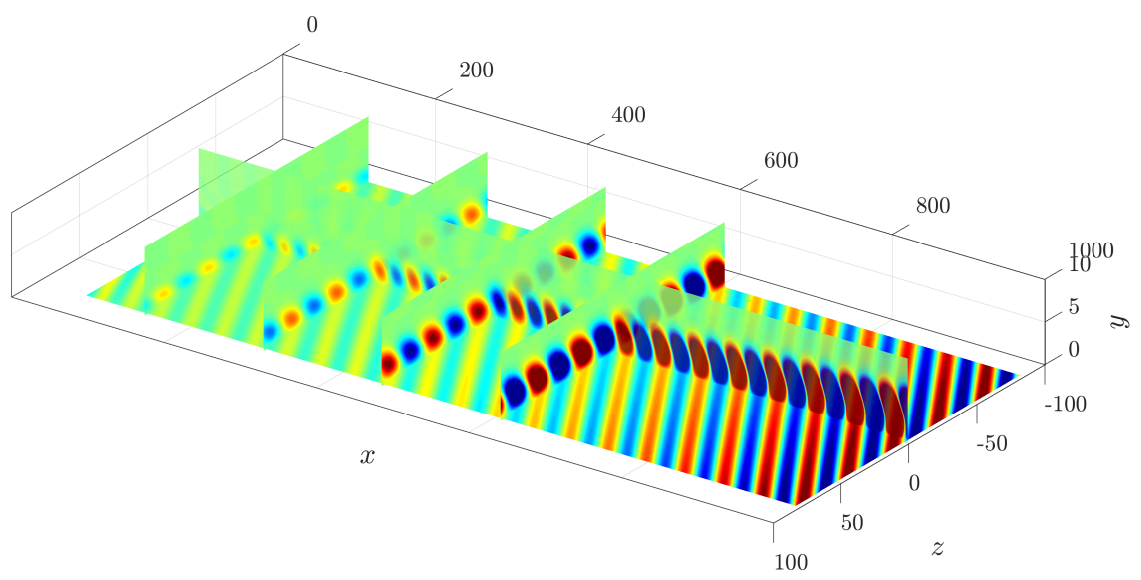

Figure 4. Three-dimensional visualization of stationary crossflow disturbance in physical space obtained from OWNS. Falkner-Skan-Cooke boundary layer with $\beta=0.19, \omega=0$. Contours of the real part of streamwise velocity perturbation $u_{s}$ along the external streamline are shown. 


\section{Conclusions}

We have extended the one-way spatial integration method to the Navier-Stokes equations for predicting the spatial stability characteristics of two- and three-dimensional wall-bounded flows. We have shown that the method can be readily used for accurate prediction of the modal transitional instability mechanisms. In this study, the method has been verified, using the Blasius and the Falkner-Skan-Cooke flows, for predicting the evolution of unstable Tollmien-Schlichting waves and crossflow vortices, respectively. The chosen test cases are validation examples where PSE performs reasonably well, but OWNS can be expected to be more general, for example handling more complex multi-wavelength modes, multiple modal instabilities with nearby eigenvalues, and optimal forcing terms, due to the accurate parabolization of the linearized operator. The low computational cost of the OWNS, compared to the one of the full equations, which is related to the ability of reducing the dimensions of the system by one by marching along a slowly evolving direction, makes the method favorable for application in fully three-dimensional flows.

\section{Appendices}

\section{A. Linearized Navier-Stokes operators}

The linearized Navier-Stokes operators in cartesian coordinates, in terms of specific volume $\nu$ and pressure $p$ are:

$$
\begin{gathered}
A=\left[\begin{array}{ccccc}
\bar{u}_{x} & -\bar{\nu} & 0 & 0 & 0 \\
0 & \bar{u}_{x} & 0 & 0 & \bar{\nu} \\
0 & 0 & \bar{u}_{x} & 0 & 0 \\
0 & 0 & 0 & \bar{u}_{x} & 0 \\
0 & \gamma \bar{p} & 0 & 0 & \bar{u}_{x}
\end{array}\right] \quad B_{1}=\left[\begin{array}{ccccc}
\bar{u}_{y} & 0 & -\bar{\nu} & 0 & 0 \\
0 & \bar{u}_{y} & 0 & 0 & 0 \\
0 & 0 & \bar{u}_{y} & 0 & \bar{\nu} \\
0 & 0 & 0 & \bar{u}_{y} & 0 \\
0 & 0 & \gamma \bar{p} & 0 & \bar{u}_{y}
\end{array}\right], \quad B_{y}=\left[\begin{array}{ccccc}
\bar{u}_{z} & 0 & 0 & -\bar{\nu} & 0 \\
0 & \bar{u}_{y} & 0 & 0 & 0 \\
0 & 0 & \bar{u}_{z} & 0 & 0 \\
0 & 0 & 0 & \bar{u}_{z} & \bar{\nu} \\
0 & 0 & 0 & \gamma \bar{p} & \bar{u}_{z}
\end{array}\right], \\
B_{x x}=B_{y y}=B_{z z}\left[\begin{array}{ccccc}
0 & 0 & 0 & 0 & 0 \\
0 & -\frac{\bar{\nu}}{R e} & 0 & 0 & 0 \\
0 & 0 & -\frac{\bar{\nu}}{R e} & 0 & 0 \\
0 & 0 & 0 & -\frac{\bar{\nu}}{R e} & 0 \\
-\frac{\gamma \bar{p}}{R e P r} & 0 & 0 & 0 & -\frac{\gamma \bar{\nu}}{R e P r}
\end{array}\right], \quad C=\left[\begin{array}{ccccc}
-\nabla \cdot \overline{\mathbf{u}} & \frac{\partial \bar{\nu}}{\partial x} & \frac{\partial \bar{\nu}}{\partial y} & \frac{\partial \bar{\nu}}{\partial z} & 0 \\
\frac{\partial \bar{p}}{\partial x}-\frac{1}{R e} \nabla^{2} \bar{u}_{y} & \frac{\partial \bar{u}_{x}}{\partial x_{2}} & \frac{\partial \bar{u}_{x}}{\partial y} & \frac{\partial \bar{u}_{x}}{\partial z} & 0 \\
\frac{\partial \bar{p}}{\partial y}-\frac{1}{R e} \nabla^{2} \bar{u}_{x} & \frac{\partial \bar{u}_{y}}{\partial x} & \frac{\partial \bar{u}_{y}}{\partial y} & \frac{\partial \bar{u}_{y}}{\partial z} & 0 \\
\frac{\partial \bar{p}}{\partial z}-\frac{1}{R e} \nabla^{2} \bar{u}_{z} & \frac{\partial \bar{u}_{z}}{\partial x} & \frac{\partial \bar{u}_{z}}{\partial y} & \frac{\partial \bar{u}_{z}}{\partial z} \\
-\frac{\gamma}{R e P r} \nabla^{2} \bar{p} & \frac{\partial \bar{p}}{\partial x} & \frac{\partial \bar{p}}{\partial y} & \frac{\partial \bar{p}}{\partial z} & \gamma \nabla \cdot \overline{\mathbf{u}}-\frac{\gamma}{R e P r} \nabla^{2} \bar{\nu}
\end{array}\right]
\end{gathered}
$$

\section{Acknowledgments}

G.R. and T.C. acknowledge the support of the Boeing Company through a Strategic Research and Development Relationship Agreement CT-BA-GTA-1.

\section{References}

\footnotetext{
${ }^{1}$ Sinha, A., Rodríguez, D., Brès, G. A., and Colonius, T., "Wavepacket models for supersonic jet noise," Journal of Fluid Mechanics, Vol. 742, 2014, pp. 71-95.

${ }^{2}$ Beneddine, S., Sipp, D., Arnault, A., Dandois, J., and Lesshafft, L., "Conditions for validity of mean flow stabilityanalysis," Journal of Fluid Mechanics, Vol. 798, 007 2016, pp. 485-504.

${ }^{3}$ Schmidt, O. T., Towne, A., Colonius, T., Cavalieri, A., Jordan, P., and Brès, G. A., "Wavepackets and trapped acoustic modes in a Mach 0.9 turbulent jet: a global stability analysis," submitted to Journal of Fluid Mechanics, 2016.

${ }^{4}$ Theofilis, V., "Global linear instability," Annual Review of Fluid Mechanics, Vol. 43, 2011, pp. 319-352.

${ }^{5}$ Herbert, T., "Parabolized stability equations," Annual Review of Fluid Mechanics, Vol. 29, No. 1, 1997, pp. 245-283.

${ }^{6}$ Bertolotti, F. P., Herbert, T., and Spalart, P. R., "Linear and nonlinear stability of the Blasius boundary layer," Journal of Fluid Mechanics, Vol. 242, 1992, pp. 441-474.

${ }^{7}$ Paredes, P., Hanifi, A., Theofilis, V., and Henningson, D. S., "The nonlinear PSE-3D concept for transition prediction in Flows with a Single Slowly-Varying Spatial Direction," Procedia IUTAM, Vol. 14, 2015, pp. 36-44.

${ }^{8}$ Towne, A. and Colonius, T., "One-way spatial integration of hyperbolic equations," Journal of Computational Physics, Vol. 300, 2015, pp. 844-861.
} 
${ }^{9}$ Towne, A. and Colonius, T., "Continued development of the one-way Euler equations: application to jets," 20th AIAA/CEAS Aeroacoustics Conference, AIAA Paper, Vol. 2903, 2014.

${ }^{10}$ Towne, A. and Colonius, T., "Improved parabolization of the Euler equations," 19th AIAA/CEAS Aeroacoustics Conference, AIAA Paper, Vol. 2171, 2013.

${ }^{11} \mathrm{Li}$, F. and Malik, M. R., "On the nature of PSE approximation," Theoretical and Computational Fluid Dynamics, Vol. 8, No. 4, 1996, pp. 253-273.

${ }^{12}$ Andersson, P., Henningson, D. S., and Hanifi, A., "On a stabilization procedure for the parabolic stability equations," Journal of Engineering Mathematics, Vol. 33, No. 3, 1998, pp. 311-332.

${ }^{13}$ Appelö, D. and Colonius, T., "A high-order super-grid-scale absorbing layer and its application to linear hyperbolic systems," Journal of Computational Physics, Vol. 228, No. 11, 2009, pp. 4200-4217.

${ }^{14}$ Thompson, K. W., "Time dependent boundary conditions for hyperbolic systems," Journal of Computational Physics, Vol. 68, No. 1, 1987, pp. 1-24.

${ }^{15}$ Poinsot, T. J. and Lelef, S. K., "Boundary conditions for direct simulations of compressible viscous flows," Journal of Computational Physics, Vol. 101, No. 1, 1992, pp. 104-129.

${ }^{16}$ Stewartson, K., The Theory of Laminar Boundary Layers in Compressible Fluids, Oxford University Press, 1964.

${ }^{17}$ Saric, W. S., Reed, H. L., and White, E. B., "Stability and transition of three-dimensional boundary layers," Annual Review of Fluid Mechanics, Vol. 35, No. 1, 2003, pp. 413-440.

${ }^{18}$ Mack, L. M., "Boundary-layer linear stability theory," AGARD Rep. No. 709, 1984.

${ }^{19}$ Cooke, J. C., "The boundary layer of a class of infinite yawed cylinders," Mathematical Proceedings of the Cambridge Philosophical Society, Vol. 46, Cambridge Univ Press, 1950, pp. 645-648.

${ }^{20}$ Schrader, L. U., Brandt, L., and Henningson, D. S., "Receptivity mechanisms in three-dimensional boundary-layer flows," Journal of Fluid Mechanics, Vol. 618, 2009, pp. 209-241.

${ }^{21}$ Tempelmann, D., Hanifi, A., and Henningson, D. S., "Spatial optimal growth in three-dimensional boundary layers," Journal of Fluid Mechanics, Vol. 646, 2010, pp. 5-37.

${ }^{22}$ Tempelmann, D., Receptivity of crossflow-dominated boundary layers, Ph.D. thesis, KTH Stockholm, 2011.

${ }^{23}$ Reibert, M. S., Nonlinear stability, saturation, and transition in crossflow-dominated boundary layers, Ph.D. thesis, Arizona State University, 1996.

${ }^{24}$ Tempelmann, D., Hanifi, A., and Henningson, D. S., "Spatial optimal growth in three-dimensional compressible boundary layers," Journal of Fluid Mechanics, Vol. 704, 2012, pp. 251-279. 